\title{
A Planar Compliant Mechanism with RRP Mobilities Based on the Singularity Analysis of a 3-US Parallel Mechanism
}

\author{
Lennart Rubbert, Stéphane Caro, Pierre Renaud and Jacques Gangloff
}

\begin{abstract}
A new design method for parallel compliant mechanisms based on the singularity analysis of parallel mechanisms is presented in this paper. Here a 3US parallel mechanism is introduced and its singular configurations are analyzed with Grassmann-Cayley algebra for the design of a compliant mechanism with RRP mobilities. A novel architecture of compliant mechanism, based on a 3-UU parallel mechanism, is presented and finally its stiffness properties are analyzed with a finite element method.
\end{abstract}

Key words: Singularity, parallel mechanism, mechanism analysis, GrassmannCayley algebra, compliant mechanism, mechanism design

\section{Introduction}

In a compliant mechanism, mobilities are obtained by material deformation. Compliant mechanisms are often obtained from a single part and they exhibit a high compactness without any backlash. They are thus considered for the design of MEMS or surgical tools, where small displacements are needed with a high accuracy.

Two open problems remain in the design of such mechanisms. First, their manufacturing remains difficult. Many manufacturing processes are only adapted to planar structures, which is a strong design constraint. Second, the synthesis of compliant mechanisms is more complex than rigid-link mechanisms. Kinematics and statics of the mechanism cannot be analyzed independently [7], and stresses in the structure need to be considered. One way to design a compliant mechanism is to syn-

Lennart Rubbert · Pierre Renaud · Jacques Gangloff

University of Strasbourg, Strasbourg, France, e-mail: \{pierre.renaud,jacques.gangloff,rubbert\}@ unistra.fr

Stéphane Caro

IRCCyN/CNRS, Nantes, France, e-mail: stephane.caro@irccyn.ec-nantes.fr 


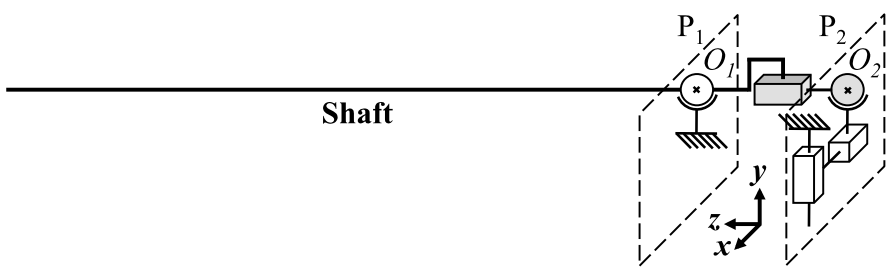

Fig. 1 Kinematic scheme of the compensation mechanism.

thesize a rigid-link mechanism and then transform the joints into compliant joints, also called flexure joints [5]. Revolute flexure joints can be easily manufactured contrary to spherical joints. The main issue during the design is therefore to identify rigid-link mechanisms that exhibit adequate kinematics and that can be transformed into compliant mechanisms.

In this paper, we propose to take advantage of singularity analysis of parallel mechanisms in order to select the architecture of a compliant mechanism. Parallel mechanisms are known for their high stiffness. We can design a compliant mechanism by considering a mechanism that exhibits a parallel singularity in a planar configuration and suppressing its actuated joints. Passive joints are then designed as flexure joints and are easy to manufacture. The obtained compliant mechanism exhibits small displacements in the directions determined from its singularity analysis as well as interesting stiffness properties. Such a design approach is, to the authors' knowledge, original and is illustrated in this paper by focusing on the design of a RRP compliant mechanism from a 3-US parallel mechanism, in the context of the design of a surgical tool.

The need for a RRP compliant mechanism in the design of a surgical tool is briefly described in Section 2. The singularity of the 3-US mechanism are analyzed with Grassmann-Cayley algebra in Section 3. The design and numerical simulation of the obtained compliant mechanism are shown in Section 4. Finally, conclusions and perspectives are written in Section 5.

\section{Need for a RRP Compliant Mechanism}

A cardiac stabilizer [2] is a surgical device that must actively immobilize the surface of a beating heart during surgery. It is mainly composed of a shaft that enters the body to reach the surface of the heart at one end. At the other end, the shaft is actuated with a mechanism which takes advantage of the oblong geometry of the shaft in order to maximize the compactness [3]. The kinematic scheme represented in Fig. 1 is considered. The shaft is controlled using a XY-stage located in a plane $P_{2}$ perpendicular to the shaft. The shaft is linked to the XY-stage with a spherical joint in $\mathrm{O}_{2}$ and oriented with a spherical joint in $O_{1}$. A prismatic joint is however needed to take into account the distance variation between $O_{1}$ and $O_{2}$. As the rota- 

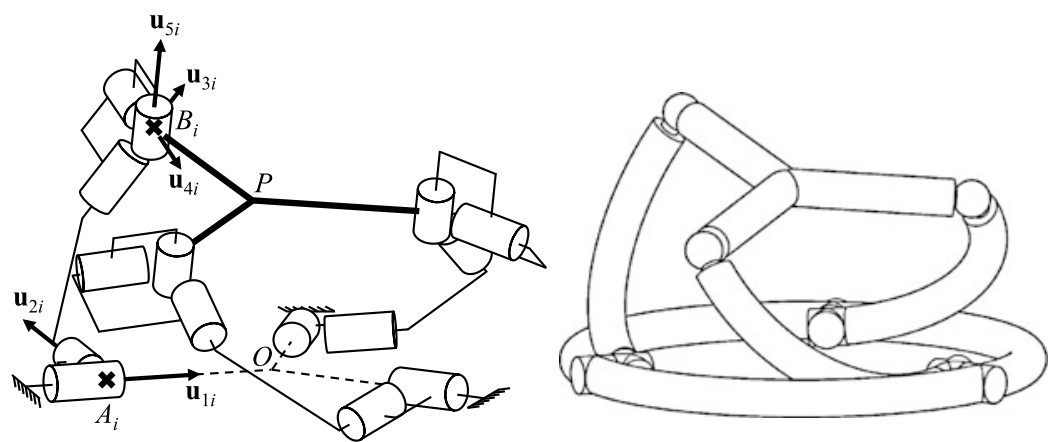

Fig. 2 Equivalent kinematic chains and architecture of the 3-US.

tion around the shaft axis is not necessary, a RRP mechanism, in grey in Fig. 1, is therefore considered to connect the shaft to the base.

\section{Singularity Analysis with Grassmann-Cayley Algebra}

The 3-US parallel mechanism has three identical legs where each leg is composed of a universal joint and a spherical joint. The first revolute joint axes of the universal joints are in the plane of the base and intersect in point $\mathrm{O}$ (Fig. 2). The 3-US mechanism has three degrees of freedom and its platform velocity can be described by three translational velocities and three angular velocities. In fact, the platform motions are coupled except when the platform is in the plane of its base. In this configuration the motion capabilities are decoupled in two angular velocities around the orthogonal in-plane axes and in one translational velocity along the axis normal to the base. In this configuration we get exactly the desired RRP motion capabilities described in Section 2.

The universal joint is similar to two revolute joints with perpendicular and intersecting axes. The spherical joint can be seen as three revolute joints with perpendicular and intersecting axes as shown in Fig. 2. This section aims to analyze the singularities of the 3-US for its five symmetrical actuation modes and the instantaneous gained motions in the vicinity of the singular configurations. The $i$ th joint of each leg is actuated for the $i$ th actuation mode of the mechanism, $i=1, \ldots, 5$. It is noteworthy that the architecture of the compliant mechanism will be directly related to the selected actuation scheme. The singularities of the 3-US are analyzed with the Grassmann-Cayley Algebra (GCA) [1, 4]. In the following, the singularity analysis for the first actuation mode is detailed as an illustrative example and the instantaneous gained motions are described for all actuation modes. 


\subsection{Analysis of the First Actuation Mode}

Twist System of the 3-US: The twist system $\mathrm{T}^{i}$ associated with the $i$ th leg of the 3-US is spanned by five zero-pitch twists defined as:

$$
\begin{array}{ll}
\hat{\varepsilon}_{01}^{i}=\left[\begin{array}{c}
\mathbf{u}_{1 i} \\
\mathbf{a}_{i} \times \mathbf{u}_{1 i}
\end{array}\right], \quad \hat{\varepsilon}_{02}^{i}=\left[\begin{array}{c}
\mathbf{u}_{2 i} \\
\mathbf{a}_{i} \times \mathbf{u}_{2 i}
\end{array}\right], \quad \hat{\varepsilon}_{03}^{i}=\left[\begin{array}{c}
\mathbf{u}_{3 i} \\
\mathbf{b}_{i} \times \mathbf{u}_{3 i}
\end{array}\right] \\
\hat{\varepsilon}_{04}^{i}=\left[\begin{array}{c}
\mathbf{u}_{4 i} \\
\mathbf{b}_{i} \times \mathbf{u}_{4 i}
\end{array}\right], \quad \hat{\varepsilon}_{05}^{i}=\left[\begin{array}{c}
\mathbf{u}_{5 i} \\
\mathbf{b}_{i} \times \mathbf{u}_{5 i}
\end{array}\right], \quad i=1,2,3
\end{array}
$$

$\mathbf{u}_{1 i}$ and $\mathbf{u}_{2 i}$ are the unit vectors of the first and second revolute joint axes of the universal joint of the $i$ th leg. $\mathbf{u}_{3 i}, \mathbf{u}_{4 i}$ and $\mathbf{u}_{5 i}$ are the unit vectors of the revolute joints associated with the spherical joint of the ith leg. $\mathbf{a}_{i}$ and $\mathbf{b}_{i}$ are the Cartesian coordinate vectors of points $A_{i}$ and $B_{i}$ shown in Fig. 2. The twist system T of the 3-US is the intersection of $\mathrm{T}^{1}, \mathrm{~T}^{2}$ and $\mathrm{T}^{3}$.

Wrench System of the 3-US: In a non-singular configuration, the constraint wrench system $\mathrm{W}^{c}$ of the 3 -US is a three-system spanned by the following three pure forces:

$$
\hat{\mathscr{F}}_{i}^{c}=\left[\begin{array}{c}
\mathbf{n}_{i} \\
\mathbf{b}_{i} \times \mathbf{n}_{i}
\end{array}\right], \quad i=1,2,3
$$

$\mathbf{n}_{i}$ being the unit vector of $\overrightarrow{A_{i} B_{i}}$. In turn, the actuation wrench system $\mathrm{W}^{a}$ of the 3US depends on its actuation scheme. In case the first revolute joint of each leg is actuated, $\mathrm{W}^{a}$ is spanned by the following three pure forces:

$$
\hat{\mathscr{F}}_{1 i}^{a}=\left[\begin{array}{c}
\mathbf{u}_{2 i} \\
\mathbf{b}_{i} \times \mathbf{u}_{2 i}
\end{array}\right], \quad i=1,2,3
$$

In case the second revolute joint of each leg is actuated, $\mathrm{W}^{a}$ is spanned by the following three pure forces:

$$
\hat{\mathscr{F}}_{2 i}^{a}=\left[\begin{array}{c}
\mathbf{u}_{1 i} \\
\mathbf{b}_{i} \times \mathbf{u}_{1 i}
\end{array}\right], \quad i=1,2,3
$$

In case the $j$ th revolute joint of each leg is actuated, $j=3,4,5, \mathrm{~W}^{a}$ is spanned by the following three pure forces:

$$
\hat{\mathscr{F}}_{j i}^{a}=\left[\begin{array}{c}
\mathbf{v}_{j i} \\
\mathbf{c}_{j i} \times \mathbf{v}_{j i}
\end{array}\right], \quad i=1,2,3
$$

$\mathbf{v}_{j i}$ being the unit vector of the intersection line $\mathscr{L}_{j i}$ of planes $\mathscr{P}_{1 i}$ and $\mathscr{P}_{j i}$. $\mathbf{c}_{j i}$ is the Cartesian coordinate vector of any point $C_{j i}$ on line $\mathscr{L}_{j i}$. $\mathscr{P}_{1 i}$ is spanned by vectors $\mathbf{u}_{1 i}$ and $\mathbf{u}_{2 i}$ and passes through point $A_{i} . \mathscr{P}_{3 i}$ is spanned by vectors $\mathbf{u}_{4 i}$ and $\mathbf{u}_{5 i}$ and passes through point $B_{i} . \mathscr{P}_{4 i}$ is spanned by vectors $\mathbf{u}_{3 i}$ and $\mathbf{u}_{5 i}$ and passes through point $B_{i} . \mathscr{P}_{5 i}$ is spanned by vectors $\mathbf{u}_{3 i}$ and $\mathbf{u}_{4 i}$ and passes through point $B_{i}$. 


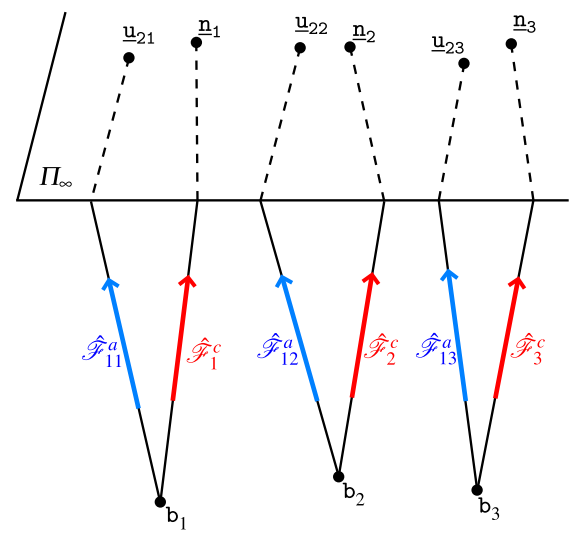

Fig. 3 Wrench graph of the 3-US associated with its first actuation mode.

As a result, the global wrench system $\mathrm{W}_{3 U S}^{k}$ of the 3-US associated with its $k$ th actuation scheme is spanned by $\mathrm{W}^{a}$ and $\mathrm{W}^{c}$, namely,

$$
\mathrm{W}_{3 U S}^{k}=\operatorname{span}\left(\hat{\mathscr{F}}_{1}^{c}, \hat{\mathscr{F}}_{2}^{c}, \hat{\mathscr{F}}_{3}^{c}, \hat{\mathscr{F}}_{k 1}^{a}, \hat{\mathscr{F}}_{k 2}^{a}, \hat{\mathscr{F}}_{k 3}^{a}\right), \quad k=1, \ldots, 5
$$

Wrench Graph of the 3-US: The six forces $\hat{\mathscr{F}}_{1}^{c}, \hat{\mathscr{F}}_{2}^{c}, \hat{\mathscr{F}}_{3}^{c}, \hat{\mathscr{F}}_{11}^{a}, \hat{\mathscr{F}}_{12}^{a}$ and $\hat{\mathscr{F}}_{13}^{a}$ form a basis of the global wrench system $W_{3 U S}^{1}$. Those wrenches are represented by six finite lines in $P_{3}$. To obtain the six extensors of the superbracket, we have to select twelve projective points on the six projective lines, i.e., two points on each line. Let $\mathrm{b}_{1}, \mathrm{~b}_{2}, \mathrm{~b}_{3}$ be the intersection points of $\hat{\mathscr{F}}_{11}^{a}$ and $\hat{\mathscr{F}}_{1}^{c}, \hat{\mathscr{F}}_{12}^{a}$ and $\hat{\mathscr{F}}_{2}^{c}, \hat{\mathscr{F}}_{13}^{a}$ and $\hat{\mathscr{F}}_{3}^{c}$, respectively. $\hat{\mathscr{F}}_{11}^{a}, \hat{\mathscr{F}}_{1}^{c}, \hat{\mathscr{F}}_{12}^{a}, \hat{\mathscr{F}}_{2}^{c}, \hat{\mathscr{F}}_{13}^{a}$ and $\hat{\mathscr{F}}_{3}^{c}$ intersect the infinite plane $\Pi_{\infty}$ at points $\underline{\mathrm{u}}_{21}=\left(\mathbf{u}_{21}, 0\right)^{T}, \underline{\mathrm{n}}_{1}=\left(\mathbf{n}_{1}, 0\right)^{T}, \underline{\mathrm{u}}_{22}=\left(\mathbf{u}_{22}, 0\right)^{T}, \underline{\mathrm{n}}_{2}=\left(\mathbf{n}_{2}, 0\right)^{T}, \underline{\mathrm{u}}_{23}=\left(\mathbf{u}_{23}, 0\right)^{T}$ and $\underline{\mathrm{n}}_{3}=\left(\mathbf{n}_{3}, 0\right)^{T}$, respectively. The wrench graph of the 3-US corresponding to its first actuation mode is illustrated in Fig. 3.

Superbracket of the 3-US: The expression of the superbracket of the 3-US associated with its first actuation mode is derived from the nine projective points depicted in Fig. 3 and takes the form: $\left[b_{1} \underline{u}_{21} b_{1} \underline{n}_{1} b_{2} \underline{u}_{22} b_{2} \underline{n}_{2} b_{3} \underline{u}_{23} b_{3} \underline{n}_{3}\right]$. This expression can be developed into a linear combination of 24 bracket monomials [4], each one being the product of three brackets of four projective points. The superbracket was simplified by using a novel graphical user interface: ${ }^{1}$

$$
\begin{array}{r}
{\left[\mathrm{b}_{1} \underline{\mathrm{u}}_{21} \mathrm{~b}_{1} \underline{\mathrm{n}}_{1} \mathrm{~b}_{2} \underline{\mathrm{u}}_{22} \mathrm{~b}_{2} \underline{\mathrm{n}}_{2} \mathrm{~b}_{3} \underline{\mathrm{u}}_{23} \mathrm{~b}_{3} \underline{\mathrm{n}}_{3}\right]=\left[\mathrm{b}_{1} \underline{\mathrm{u}}_{21} \underline{\mathrm{n}}_{1} \dot{\mathrm{b}}_{2}\right]\left[\mathrm{b}_{1} \mathrm{~b}_{2} \underline{\mathrm{n}}_{2} \mathrm{~b}_{3}\right]\left[\underline{\dot{u}}_{22} \underline{\mathrm{u}}_{23} \mathrm{~b}_{3} \underline{\mathrm{n}}_{3}\right]} \\
=\left(\mathrm{b}_{1} \underline{\mathrm{u}}_{21} \underline{\mathrm{n}}_{1}\right) \wedge\left(\mathrm{b}_{2} \underline{\mathrm{u}}_{22} \underline{\mathrm{n}}_{2}\right) \wedge\left(\mathrm{b}_{3} \underline{\mathrm{u}}_{23} \underline{\mathrm{n}}_{3}\right) \wedge\left(\mathrm{b}_{1} \mathrm{~b}_{2} \mathrm{~b}_{3}\right)
\end{array}
$$

\footnotetext{
${ }^{1}$ http://www.irccyn.ec-nantes.fr/ caro/SIROPA/GUIGCASiropa.jar
} 
where dotted letters stand for permuted elements and $\wedge$ denotes the meet operator [4].

Geometric Parallel Singularity Conditions: Let $\Pi_{l}$ be the plane passing through point $B_{l}$ and spanned by $\mathbf{u}_{2 l}$ and $\mathbf{n}_{l}, l=1,2,3$. Let $\Pi_{4}$ be the plane passing through points $B_{1}, B_{2}$ and $B_{3}$. Equation (8) vanishes, namely, the 3 -US reaches a parallel singularity for its first actuation mode, if and only if planes $\Pi_{1}, \Pi_{2}, \Pi_{3}$ and $\Pi_{4}$ intersect at least at one point.

\subsection{Singularity Conditions and Instantaneous Gained Motions}

Singularity conditions have been determined for the first actuation mode. A similar procedure was used to analyze the singularities associated with the second actuation mode of the 3-US. It is noteworthy that the simplification of the superbracket expression is not straightforward for the third, fourth and fifth actuation modes. Therefore, we can use GCA and Grassmann geometry as complementary approaches in order to derive the singularity conditions related to those five actuation modes as explained in [1]. In case of the third, fourth and fifth actuation modes, the Grassmann-Caley algebra is directly applied to the planar configuration when the plane of the platform normal to the $\mathbf{u}_{5 i}$ is in the plane of the base defined by $\mathbf{u}_{1 i}$ for the singularity analysis (Fig. 2).

For the five actuation modes, the 3-US turns to be singular in the planar configuration, i.e., when the base and moving-platform are coplanar. As a matter of fact, from Eq. (7), the global wrench system of the 3-US becomes a 3-system composed of six coplanar pure forces in this configuration. Its twist system $\mathrm{T}_{3 U S}$ is reciprocal to $\mathrm{W}_{3 U S}^{k}, k=1, \ldots, 5$ and becomes also a 3 -system in this configuration even if the actuated joints are locked. $\mathrm{T}_{3 U S}$ is spanned by two zero-pitch twists with non parallel axes belonging to $\Pi_{4}$ and one infinite-pitch twist of axis normal to $\Pi_{4}$. As a consequence, the instantaneous gained motions of the moving-platform correspond to the desired RRP motion, namely, one translation along the direction normal to $\Pi_{4}$ and two rotations about non parallel axes belonging to $\Pi_{4}$ for all actuation modes in the planar configuration.

\section{Design of the Compliant Mechanism}

For each actuation mode, the 3-US gains the desired RRP motion described in Section 2. Since the mechanism will be machined in a plane, it is more convenient to machine flexure joints with revolute axes in the plane. Therefore, among the five revolute joints per leg that can be suppressed, we choose to eliminate the fifth revolute joint of each leg ( $\mathbf{u}_{5 i}$, Fig. 2) which is the only one to have an axis normal "to the base and moving platform" in the planar configuration of the 3-US. Therefore, 

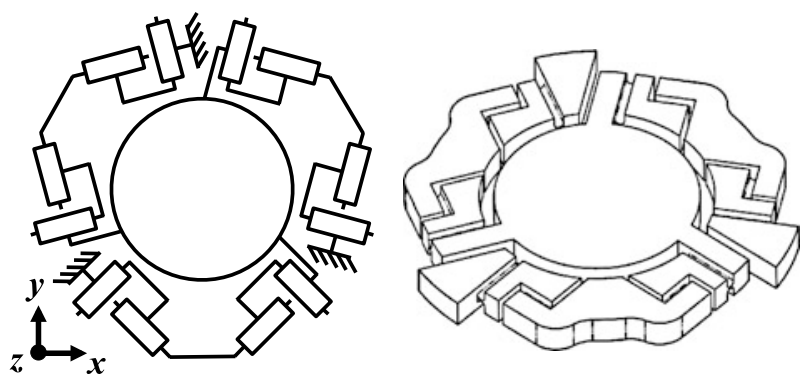

Fig. 4 Kinematic scheme and CAD view of the 3-UU compliant mechanism.

the 3-US becomes a 3-UU as illustrated in Fig. 4. The 3-UU compliant mechanism is then designed by replacing each revolute joint by a circular flexure hinge. The computer-aided design of the compliant 3-UU is shown in Fig. 4.

The compliant mechanism is assessed by computing the $6 \times 6$ compliance matrix, which relates the displacement $\mathbf{u}=\left[x ; y ; z ; \theta_{x} ; \theta_{y} ; \theta_{z}\right]$ to the load $\mathbf{L}=$ $\left[F_{x} ; F_{y} ; F_{z} ; M_{x} ; M_{y} ; M_{z}\right]$ applied at the center of the platform [6]:

$$
\mathbf{u}=\mathbf{C} . \mathbf{L}
$$

The $6 \times 6$ compliance matrix $\mathbf{C}$ is evaluated using a finite element analysis (PTC ProMechanica):

$$
\mathbf{C}=\left[\begin{array}{cccccc}
\mathbf{7 . 3 e}-5 & -2.5 \mathrm{e}-8 & -4.2 \mathrm{e}-8 & -3.4 \mathrm{e}-5 & 5.3 \mathrm{e}-8 & 1.4 \mathrm{e}-6 \\
3.8 \mathrm{e}-8 & \mathbf{7 . 3 e}-5 & -2.7 \mathrm{e}-8 & -8.0 \mathrm{e}-6 & 9.0 \mathrm{e}-6 & 1.4 \mathrm{e}-6 \\
-1.1 \mathrm{e}-7 & -5.9 \mathrm{e}-8 & \mathbf{1 . 7 e - 2} & 4.1 \mathrm{e}-5 & 1.1 \mathrm{e}-4 & 1.2 \mathrm{e}-6 \\
-1.9 \mathrm{e}-6 & 0.0 & -8.7 \mathrm{e}-5 & \mathbf{1 . 4} & -6.0 \mathrm{e}-3 & 4.1 \mathrm{e}-5 \\
0.0 & 1.5 \mathrm{e}-6 & -1.2 \mathrm{e}-5 & -8.4 \mathrm{e}-4 & \mathbf{1 . 4} & 3.0 \mathrm{e}-5 \\
0.0 & -6.0 \mathrm{e}-8 & -9.6 \mathrm{e}-7 & 4.0 \mathrm{e}-6 & 0.0 & \mathbf{6 . 9 e - 3}
\end{array}\right]
$$

The translational stiffness along the vertical axis is approximately 240 times lower than the in-plane translational stiffnesses. The rotational stiffness along the vertical axis is 210 times greater than the in-plane rotational stiffnesses. This is verified when three forces are applied at the center of the mechanism: there is only a displacement along the vertical axis (Fig. 5 left). When three moments are applied at the center of the mechanism, there are two rotations about in-plane axes (Fig. 5 right). Therefore, the compliant mechanism behaves as a RRP mechanism.

\section{Conclusion}

A new design method for parallel compliant mechanism based on singular configurations has been presented in this paper. A 3-US parallel mechanism has been 


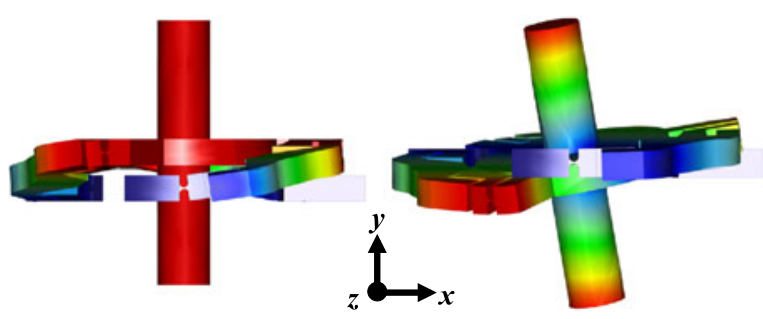

Fig. 5 Finite element analysis of the 3-UU compliant mechanism under a combined load (left) and moment (right).

introduced and based on the Grassmann-Cayley algebra it has been possible to analyze the singularities in this mechanism for the design of a compliant mechanism with RRP mobilities. The stiffnesses of the compliant mechanism have been evaluated with a finite element analysis. The high ratio between the stiffnesses in the different motion directions shows that the compliant mechanism behaves as a RRP mechanism. Hence, a new compliant planar parallel architecture for a RRP compliant mechanism has been proposed. The next step will consist in optimizing the mechanism to match the expected performances in terms of relative stiffnesses and stresses in the material.

\section{References}

1. Amine, S., Tale-Masouleh, M., Caro, S., Wenger, P., Gosselin, C.: Singularity analysis of 3T2R parallel mechanisms using Grassmann-Cayley algebra and Grassmann line geometry. Mech. Mach. Theory, (2012). doi:10.1016/j.mechmachtheory.2011.11.015

2. Bachta, W., Renaud, P., Laroche, E., Forgione, A., Gangloff, J.: Cardiolock: An active cardiac stabilizer, first in vivo experiments using a new robotized device. Comput. Aided Surg. 13(5), 243-254 (2008)

3. Bachta, W., Renaud, P., Laroche, E., Gangloff, J.: Cardiolock2: Parallel singularities for the design of an active heart stabilizer. In: ICRA, pp. 3839-3844 (2009)

4. Ben-Horin, P., Shoham, M.: Singularity analysis of a class of parallel robots based on Grassmann-Cayley algebra. Mech. Mach. Theory 41(8), 958-970 (2006)

5. Howell, L.: Compliant Mechanisms. Wiley-IEEE (2001)

6. Lobontiu, N.: Compliant Mechanisms - Design of Flexure Hinges. CRC Press (2003)

7. Rubbert, L., Renaud, P., Bachta, W., Gangloff, J.: Compliant mechanisms for an active cardiac stabilizer: Lessons and new requirements in the design of a novel surgical tool. Mech. Sci. 2, $119-127(2011)$ 